\title{
Zum Verhältnis von Reallabor, Realexperiment und Stadtplanung am Beispiel kooperativer Freiraumgestaltung
}

\author{
Charlotte Räuchle \\ Eingegangen: 11. Oktober 2020 - Angenommen: 11. März 2021 - Online veröffentlicht: 25. März 2021
}

\begin{abstract}
Zusammenfassung
Der Beitrag untersucht das Verhältnis von Reallabor und Planung und fragt nach den Chancen, die das Realexperiment der Stadtplanung bieten kann. Die empirische Grundlage liefert eine Fallstudie zur Teilhabe und zur Stärkung des sozialen Zusammenhalts durch kooperative Freiraumentwicklung in superdiversen Quartieren. Obwohl der Themenkomplex „Experiment und Planung“ im urbanen Kontext in der jüngeren Vergangenheit verstärkt Aufmerksamkeit gefunden hat, mangelt es weiterhin an theoretischen Analysen und empirischen Untersuchungen. Hier wird daher zunächst auf das Verhältnis von Experiment und Planung aus systematisch-theoretischer Perspektive eingegangen - im Hinblick auf die Ziele, die Art des Wissens, die Verfahren der Wissensproduktion, die Instrumente und die Prozesse. Dann werden anhand der Fallstudie die inhaltlichen, instrumentellen und akteurbezogenen Dimensionen dieses Verhältnisses analysiert. Im Ergebnis zeigt sich, dass Reallabore und Realexperimente der Stadtplanung durchaus Chancen eröffnen können: in inhaltlicher Hinsicht die Produktion differenzierteren Ziel-, System- und Transformationswissens, in instrumenteller Hinsicht die Bereitstellung offenerer Beteiligungsformate, in akteurbezogener Hinsicht die Bildung neuer Governance-Arrangements. Ob dies wirklich als Chance begriffen wird, hängt jedoch vom lokalen Planungsobjekt und von der lokalen Planungskultur ab.
\end{abstract}

$\bowtie$ Dr. Charlotte Räuchle, Institut für Geographische Wissenschaften, Arbeitsbereich Globalisierung, Transformation, Gender, Freie Universität Berlin, Malteserstraße 74-100, 12249 Berlin, Deutschland charlotte.raeuchle@fu-berlin.de

\section{(c) (1)} (c) 2021 Räuchle; licensee oekom verlag. This Open Access article is published under a Creative Commons Attribution 4.0 International License.
Schlüsselwörter: Reallabore - Realexperimente Freiraumentwicklung - Stadtplanung - sozialer Zusammenhalt = experimentelle Wissensproduktion

The relationship between real-world labs, realworld experiments and urban planning, using the example of cooperative open space development

\begin{abstract}
This paper examines the relationship between real-world laboratory and planning and highlights the potentials that realworld experiments offer to urban planning. It is based on a case study on cooperative open space development to unite residents and strengthen social cohesion in superdiverse neighbourhoods. Although the subject of „experiment and planning" has received increasing attention recently, there is still a lack of theoretical and empirical analysis. Therefore, this study, first, addresses the relationship between experiment and planning from a conceptual perspective - regarding objectives, types of knowledge, methods of knowledge production, instruments and processes. Second, based on the case study, the content, instrumental and actor-related dimensions of this relationship are analysed. The results show that real-world experiments can open up opportunities for urban planning - in terms of content (production of more differentiated knowledge), instruments (provision of more open participation formats), and actors (formation of new governance arrangements). Whether this is really seen as an opportunity, however, depends on the local planning object and, in particular, on the local planning culture.
\end{abstract}


Keywords: Real-world labs - Real-world experiments Open space development - Urban planning = Social cohesion Experimental knowledge production

\section{Realexperimente als neues Instrument der Stadtplanung}

Seit einigen Jahren erfährt der Themenkomplex „Experiment und Stadt" - als eine Facette des übergreifenden experimental turn in den Sozial- und Wirtschaftswissenschaften - verstärkt Aufmerksamkeit. Dies erfolgt fast zwangsläufig aus der wissenschaftlichen und praktischen Debatte um Reallabore bzw. die verschiedenen Formate des „Real-World Lab“, die die Forschungsinfrastruktur für (Real-)Experimente bereitstellen, oder geht dieser sogar voraus (Scholl/Kemp 2016: 89-91; Schäpke/Stelzer/ Bergmann et al. 2017: 28-45). Dabei wird das Experiment bereits als neuer Ansatz kommunaler Steuerung verstanden, obwohl das Verhältnis von Reallabor und lokalem Kontext noch weitgehend ungeklärt ist (Evans/Karvonen/Raven 2016: 1-6; Caprotti/Cowley 2017: 1441-1442). Immer noch mangelt es an theoretischen und empirischen Untersuchungen zum Verhältnis von Reallabor/Realexperiment zu Stadtentwicklung/Stadtplanung. Einerseits wird die ,absence of experiments in planning" (Honey-Rosés/Stevens 2019: 267) konstatiert: Grundsätzlich sei unklar, ob über die in der Stadtforschung und Stadtentwicklung vorhandenen experimentellen Ansätze überhaupt weitreichendere Wirkungen erzielt werden könnten. Andererseits werden „city labs as vehicles for innovation in urban planning processes“ (Scholl/Kemp 2016: 89) gesehen. Experimentelle Formate unter Einsatz partizipativer und aktivierender Methodenmixe seien gang und gäbe in der Planungswissenschaft und -praxis (Kanning 2018: 7-8). Diese optimistische Sichtweise geht so weit, dass ,the experiment with its co-creative dogmas seems to be a perfect fit for current governance policies in urban planning" (Jacobsen 2018: 36).

Beide Einschätzungen sind zwar durchaus miteinander kompatibel, weisen aber auf eine ungeklärte Beziehung zwischen Reallabor und Stadtplanung hin. Das erstaunt insofern, als die Stadt als Labor und das Experiment als Mittel der Stadtentwicklung und -planung eine lange Tradition haben, die bis Anfang des letzten Jahrhunderts zurückreicht (Schneidewind 2014: 3). Damals wurden allerdings ,Labor ${ }^{\circ}$ und ,Experiment" eher in einem metaphorischen Sinn verwendet, um Städte als die Orte zu beschreiben, an denen aufgrund sozialer Dichte quasi automatisch ,getestet " wurde, unter welchen Bedingungen welche Formen sozialen Zusammenlebens möglich waren. Heute wird dagegen das (Real-)Experiment als eine Methode der Intervention und Wissensproduktion in der interdisziplinären Stadtforschung und Planungswissenschaft verstanden, das aber noch nicht präzise definiert sei (Karvonen/van Heur 2014: 380; Scholl/ Kemp 2016: 89). In der deutschsprachigen Debatte wird zwar immer wieder aus der Planungspraxis heraus auf die Nähe des Experiments zu etablierten Instrumenten im Rahmen der Nationalen Stadtentwicklungspolitik wie beispielsweise Internationale Bauausstellungen und Modellvorhaben (Altrock 2014: 29; Kanning 2018: 8) und aus der Planungstheorie heraus auf Ähnlichkeiten wie etwa zum performativen Planungsideal verwiesen (Altrock 2014: 24; KarowKluge 2008: 87-88). Es bleibt jedoch auszuloten, wie sich das Verhältnis von Reallabor/Realexperiment und Stadtplanung in Theorie und Praxis genau darstellt.

Am Beispiel des Reallabor-Projekts „KoopLab: Teilhabe durch kooperative Freiraumentwicklung in Ankunftsquartieren" (KoopLab) ${ }^{1}$ werden im Beitrag daher folgende Fragen diskutiert: Gibt es und, wenn ja, in welcher Hinsicht Unterschiede zwischen Stadtplanung und Reallabor/ Realexperiment? Eröffnen sich mit dem Realexperiment als Instrument neue Möglichkeiten für die Stadtplanung?

Der Beitrag ist wie folgt aufgebaut: Zunächst wird auf den systematisch-theoretischen Zusammenhang von Stadtplanung, Reallabor und Realexperiment näher eingegangen (Kapitel 2), bevor die Fallstudie sowie der methodische $\mathrm{Zu}$ griff vorgestellt werden (Kapitel 3). Im dann folgenden Kapitel werden empirische Befunde aus dem Reallabor-Projekt „KoopLab“ und seinen Realexperimenten auf ihr Verhältnis zur Stadtplanung hin befragt, und zwar bezüglich der Dimensionen „Inhalte“ (Kapitel 4), „Instrumente“ (Kapitel 5) sowie „Governance“ (Kapitel 6). Abschließend wird noch einmal der Frage nachgegangen, ob das Realexperiment potenzielle Chancen für die Stadtplanung bieten kann (Kapitel 7).

\section{Zum Verhältnis von Reallabor, Realexperiment und Stadtplanung aus systematisch-theoretischer Perspektive}

Zwar kann man Reallabore zumindest in der Stadtforschung inzwischen als etablierte Instrumente der Stadtentwicklungspolitik ansehen - dies schlägt sich auch in entsprechenden Förderpolitiken nieder -, allerdings steht ein systematisch-theoretischer Zugriff auf das Verhältnis von Realexperiment und Stadtplanung noch aus. Wenn im Folgenden der Begriff „Experiment“ verwendet wird, ist damit der methodische Zugriff dieser Form der Wis-

1 https://www.kooplab.de (03.03.2021). 
sensproduktion ganz allgemein gemeint, während Realexperiment die spezifische Anwendung im Rahmen des Reallabors bezeichnet. Des Weiteren werden die Begriffe „Realexperiment“ und „Intervention“ synonym verwendet (vgl. auch Defila/Di Giulio 2018: 22-24).

\subsection{Realexperiment}

Um die Charakteristika des Realexperiments genauer herauszuarbeiten, lohnt sich ein Vergleich mit dem Laborexperiment als Referenzpunkt (vgl. zum Folgenden Parodi/Beecroft/Albiez et al. 2016: 15-16; Beecroft/Trenks/ Rhodius et al. 2018: 75-96; Puttrowait/Dietz/Gantert et al. 2018: 195-202). Ein Realexperiment ist dann durch folgende Merkmale gekennzeichnet: (1) Es ist in einem spezifischen Kontext räumlicher und physischer, sozialer, ökonomischer und politischer, letztlich gesellschaftlicher Realität angesiedelt und damit deren ganzer Komplexität ausgesetzt. Seine Prozesse sind dadurch im Vergleich zu denen im Laborexperiment schwerer zu kontrollieren. (2) Die Prozesse im Realexperiment können wie die im Laborexperiment zwar wiederholt werden, aber die permanent sich verändernden Kontexte machen die Beobachtung der UrsacheWirkung-Zusammenhänge zwischen abhängigen und unabhängigen Variablen schwieriger. (3) Die transdisziplinäre Ausrichtung des Realexperiments verlangt im Vergleich zu der des Laborexperiments ein Ko-Design der Versuchsanordnung und eine Ko-Produktion des Wissens mit den beteiligten Akteuren der Zivilgesellschaft, Kommunalverwaltung, Wirtschaft etc. (4) Das bedeutet zugleich eine kontinuierliche methodische Reflexion des Forschungsprozesses mit allen Beteiligten und entsprechenden Eingriffen in das laufende Realexperiment. Das Reallabor ist somit experimentell-reflexiv, rekursiv-lernend konzipiert. (5) Der Grad der Verallgemeinerung der Ergebnisse ist angesichts der Vielzahl der kontextspezifischen Faktoren deutlich begrenzter als beim Laborexperiment. (6) Zudem dient das Realexperiment als Instrument des Reallabors, das per se transformative Ziele verfolgt, bewusst dem gesellschaftlichen Wandel. Das Realexperiment soll im Rahmen des Reallabors handlungsleitendes Transformationswissen erzeugen, um normative Ziele zu erreichen.

Dies ist eine idealtypische Kennzeichnung. Ob bei so starken Abweichungen vom Laborexperiment der Begriff des „Experiments“ überhaupt zutreffend ist, sei dahingestellt. Allenfalls stellt das Realexperiment eine hybride Form des Experiments dar, weil es sich zwischen Wissenserzeugung und Wissensanwendung, kontrollierten und unkontrollierten Rahmenbedingungen, die Umwelt beschreibenden bzw. erklärenden und verändernden Zielen bewegt (Schneidewind 2014: 2). In jedem Fall wird seine inflationäre und häufig unreflektierte Verwendung in den
Sozialwissenschaften seit Langem kritisiert (Karvonen/van Heur 2014: 379-381; May/Perry 2016: 33-36). In seiner realtypischen Ausprägung ist das Realexperiment vielfältigen Imponderabilien und Restriktionen ausgesetzt, die zu erheblichen Abweichungen von dieser idealtypischen Kennzeichnung führen können.

\subsection{Planung und Realexperiment}

,Planung' ist zunächst einmal das Gegenteil von Experiment. Planung bedeutet auf der Basis von sicherem Wissen über den Ist-Zustand, über das festgesetzte Ziel und über die Wirkung der eingesetzten Instrumente endgültige, haftungsvermeidende, risikoaverse Entscheidungen im Sinne eines Masterplans über zukünftiges Handeln zu treffen. Sie soll Handlungssicherheit schaffen. Wenn ein Plan genehmigt ist und mit seiner Umsetzung begonnen wird, endet die Planung. Auch dies ist eine idealtypische Beschreibung, die mit der realtypischen Ausprägung durchaus nicht übereinstimmen muss (Fürst 2018: 1712-1713).

Das Verhältnis von Realexperiment und Planungspraxis hängt jedenfalls wesentlich vom Planungsverständnis bzw. von der Planungskultur ab. Geht man von einem rationalstringenten Planungsverständnis bzw. technokratisch-hierarchischem Planungsansatz aus, dürfte die transdisziplinäre und reflexive Dimension der Planung wenig entwickelt sein und das Realexperiment nur schwer einen Platz in dieser Art von Planungsarchitektur finden. Überschaut man die Planungsliteratur der jüngeren Vergangenheit, kann allerdings der Eindruck entstehen, dass es linear-hierarchische, stringente Planungsverfahren gar nicht mehr gibt. Planung scheint nur noch aus „offenen, kommunikativen Aushandlungsprozessen und kooperativer Zusammenarbeit unterschiedlicher Akteure in netzwerkartigen Strukturen“ zu bestehen (Danielzyk/Sondermann 2018: 964; KarowKluge 2008: 73-85; Knieling 2018: 1230-1231). Im modernen Verständnis besitzt Planung jedenfalls eine „hohe Flexibilität durch eine situationsgerechte Orientierung auf spezifische Herausforderungen und entsprechend angepasste Verfahren und Instrumente" (Dorstewitz 2014: 433). Manche Theoretiker/-innen, aber auch Praktiker/-innen modellieren Stadtplanung entsprechend dem kritisch-rationalistischen Falsifikationskriterium gar als Versuch-IrrtumVerfahren, in dem sich der Plan als „Hypothese“ und die Planumsetzung als „Experiment“ in einem fortlaufenden Rückkoppelungsprozess befinden (Dorstewitz 2014: 433; Deutscher Städtetag 2013: 5-11). Von flexiblen und offenen Steuerungskreisläufen ist ebenso die Rede wie von inkrementellen, emergenten Planungsprozessen. Begrifflich, aber auch inhaltlich verschmelzen Experiment und Planung, wenn von ,performativer Planung“ die Rede ist und performativ und experimentell synonym verwendet werden 
(Altrock 2014: 21-26). Diese Ausrichtungen bedeuten nun freilich nicht, dass sich Planung nur noch auf Moderation beschränkt. Planungsprozesse sollen durchaus strategisch und inhaltlich auf planerische Vorgaben hin angelegt sein.

Dennoch ergibt sich ,theoretisch“ und ,praktisch “ eine erhebliche Nähe zwischen Realexperiment und solcher Art von offener, kooperativer Planungspraxis. Allerdings hängt diese entscheidend vom Planungsprojekt ab. Jedenfalls sind Experimente reversibel und nicht auf Dauer angelegt. Sie nutzen den Raum eben nur temporär. Sozial-partizipative Experimente im Besonderen stellen zeitlich befristete Veränderungen des sozialen Handlungskontextes dar, um etwas über den Raum und seine Nutzung zu erfahren. Sie sind keine ,Vorwegnahme einer künftigen Gestalt [...] durch rationale Nutzung verfügbaren Wissens in einem Plan“ (Schäfers 1992: 232). Grundsätzlich kommen sie einer ,Planungskultur' entgegen, die stärker als technokratisch-hierarchische Planung subjektive Werthaltungen und Wahrnehmungsmuster, Intuitionen und Traditionen berücksichtigt und zwar vornehmlich im lokalen, kleinräumigen Kontext (Sondermann 2017: 45-47; Othengrafen/Reimer 2018: 1734-1735). Es bleibt allerdings der entscheidende Unterschied, dass die Planung ein bestimmtes Ziel verfolgt, das Experiment dagegen (kausale) Beziehungen aufzeigen soll. Erst im Rahmen des Reallabors ist das Experiment in der Form des Realexperiments transformativ angelegt.

\subsection{Reallabor und Stadtplanung}

\subsubsection{Ziele}

Vor dem oben genannten Hintergrund liegen das städtische Reallabor und die städtische Planung so weit nicht auseinander. Beide sind auf Transformation angelegt - wobei die Transformationsdebatte für die Planungswissenschaften relativ neu ist - , verfolgen normative Ziele und laden den urbanen Raum entsprechend vorherrschender, ,hegemonialer' Paradigmen mit Bedeutung auf, weil sie zwar von seinen baulichen Strukturen ausgehen, ihn gleichzeitig aber als soziale Konstruktion begreifen. Die normativen Ziele, die im für die Planung relevanten Recht vorgegeben sind, entsprechen denen in den politischen Förderprogrammen, die wiederum für die Reallabore wichtig sind: Nachhaltigkeit, ökologischer Stadtumbau, sozialer Zusammenhalt, Integration und weitere. Aber auch bei konkreteren Projekten der Planung und bei Interventionen der Reallabore, das heißt bei der Operationalisierung übergeordneter Werte und Leitbilder, dürften sie sich ausgesprochen ähnlich sein. Stadtentwicklungskonzepte unterliegen dem Zeitgeist, denen sich weder die Reallaborakteure noch die Stadtplaner/-innen entziehen können. Letztlich hängt es natürlich vom einzelnen Reallabor und einzelnen Planungsprojekt ab, welche konkreten Werte und Normen verwirklicht werden sollen. Die
Themen „soziale Stadt“, „soziale Kohäsion“, „Integration“ sind jedenfalls Querschnittsthemen, die sich keiner Fachplanung zuordnen lassen. Berücksichtigt man neben dieser inhaltlichen Dimension zusätzlich die - wenn man so will - ,funktionale', so verfolgen Reallabore Forschungs-, Praxis- und Bildungsziele (Beecroft/Trenks/Rhodius et al. 2018: 79-83; Defila/Di Giulio 2018: 11), Planung dagegen widmet sich vornehmlich Praxiszielen (Kanning 2018: 15).

\subsubsection{Art des Wissens}

Neben dem Wissen über den urbanen Kontext des Reallabors (Systemwissen) und seine normativen Ziele (Zielwissen) soll das Reallabor mit seinen Realexperimenten also Wissen über den Weg zum Erreichen der Ziele produzieren (Transformationswissen) (ProClim 1997: 15; Beecroft/ Trenks/Rhodius et al. 2018: 79). Das Experiment per se erzeugt, wie erwähnt, lediglich deskriptives und (eventuell) kausales Wissen. Es schafft allerdings nie sicheres Wissen. Die ihm meist explizit, zumindest aber implizit zugrundeliegenden (Hypo-)Thesen lassen sich aus kritisch-rationalistischer Sicht grundsätzlich nicht beweisen (verifizieren), sondern nur widerlegen (falsifizieren). Auch Realexperimente müssen jedenfalls im Hinblick auf das Ergebnis offen angelegt sein, bergen ein hohes $\mathrm{Ma}$ an Unsicherheit in sich und gehen davon aus, dass sich auch mit Unsicherheit produktiv arbeiten lässt. ,Erfolg' - wie immer auch definiert - wird nicht garantiert. Allerdings kann auch der ,Misserfolg ' (learning by failing) bzw. die ,Überraschung “ verwertbares Wissen produzieren. Letztlich schafft das Experiment ,sicheres“ Unwissen. Es kann als „Metapher des bewussten Umgangs mit Nichtwissen" verstanden werden (Groß 2017: 21). Dieses induktiv gewonnene Wissen soll dann so aufbereitet werden, dass es verallgemeinert und im Rahmen des Reallabors transformativ eingesetzt werden kann.

Dies gilt letztlich auch für die Planung. Sie erfolgt fast immer unter Unsicherheit, sobald im Rahmen des Systemwissens neben materiellen, baulichen Strukturen auch immaterielle, soziale Tatbestände erfasst werden sollen (Abbott 2005: 238-240). Indem ein Plan die zukünftige Entwicklung prognostiziert, vereinfacht er sie zugleich, sodass angesichts der Unwägbarkeiten der Zukunft auch das Zielwissen unsicher ist. Schließlich arbeitet Planung ebenfalls mit unsicherem Transformationswissen, weil die Wirkungen seiner Instrumente nicht genau abgeschätzt werden können. Das im Rahmen ,moderner' Planung partizipativ, kooperativ unter Berücksichtigung der besonderen Wahrnehmungsmuster, Traditionen und Meinungen vor Ort erzeugte Wissen ähnelt durchaus dem reallaborbasierten. Beide wollen das so hoch gehandelte ,sozial robuste Wissen ' generieren. Beide gehen davon aus, dass Wissen sozial konstruiert 
wird und zwar von bestimmten gesellschaftlichen Gruppen in bestimmten gesellschaftlichen Kontexten.

\subsubsection{Methoden der Wissensproduktion}

Die Methoden der Wissensproduktion im Reallabor und in der Stadtplanung ähneln und unterscheiden sich zugleich. Der Ansatz des Reallabors greift einerseits auf den Kanon traditioneller Methoden der Ingenieur-, Natur-, Geistesund Sozialwissenschaften zurück, andererseits kombiniert er diese auf ungewohnte Weise und entwickelt neue - so zumindest der Anspruch (Schneidewind 2014: 4). Es geht dabei vor allem darum, unterschiedliche Wissensbestände aufzudecken und die dafür notwendige Erhebung und Auswertung des Materials - die verschiedenen Arten erfordern unterschiedliche Methoden - vorzunehmen sowie die dafür notwendige Kommunikation - wiederum sind dafür unterschiedliche Methoden in mehr oder weniger großen und heterogenen Gruppen zu nutzen - zu organisieren (Defila/Di Giulio 2018: 22-24). Mit den Methoden des Realexperiments wird praktisches Transformationswissen erzeugt. Die damit zusammenhängenden methodischen Herausforderungen beziehen sich unter anderem auf die Kontextabhängigkeit der Realexperimente bzw. das Generalisierungspotenzial der Ergebnisse und die begrenzte Kontrollierbarkeit der Rahmenbedingungen. Außerdem müssen Methoden gefunden werden, die ,echte Auseinandersetzungen zwischen den Beteiligten" möglich machen und zwar sowohl im Hinblick auf die Differenzen als auch die Gemeinsamkeiten (Defila/Di Giulio 2019: 17). Eine weitere methodische Herausforderung ist sicherlich die Zusammenführung der verschiedenen Wissensbestände auf eine Weise, die dem transformativen Anspruch des Reallabors gerecht wird. Zum Einsatz kommt also - zumindest bei primär sozial-partizipativen Reallaboren - sowohl ein Mix aus ,klassischen' sozialwissenschaftlichen Methoden als auch Ansätze, denen der Anspruch des transdisziplinären Ko-Designs und der transdisziplinären Ko-Produktion von Realexperimenten zugrunde liegen.

Die Methoden, die zur Wissensproduktion in der Planung genutzt werden, stammen bei der Ist-Analyse ebenfalls aus den Ingenieurs-, Natur- oder Sozialwissenschaften: „Planung basiert auf den Schritten Analyse, Bewertung, Zielfindung, Handlung, Evaluation. Damit sind Dimensionen des Fachwissens/Systemwissens (Analyse und Bewertung), des Orientierungswissens (normative Zielfindung) sowie des Transformationswissens (Handeln) im Planungsprozess implementiert. Der Planungsprozess zielt auf handlungsleitendes Wissen [...] und die praktische Umsetzung. Planung ist stets transformatorisch ausgerichtet. Daher lassen sich die Methoden der Transformation in der Raumplanung mit denen der transformativen Forschung in Bezug setzen“ (Hahne 2019: 52).
Auch die Stadtplanung nutzt also einen Mix aus quantitativen und qualitativen Methoden, der zum großen Teil dem des Reallabors ähnelt.

\subsubsection{Instrumente}

Grundsätzlich erfolgt die Entwicklung des städtischen Raumes erstens über planungs- und bauordnungsrechtliche Instrumente (verbindliche Rechtsvorschriften), zweitens über wirtschaftliche, tauschbasierte Instrumente (rechtskräftige, aber kündbare Verträge) und drittens über kommunikativinformative, persuasive Instrumente (überzeugende Argumente). Die rechtsförmlichen Instrumente des Allgemeinen Städtebaurechts sind für Reallabore insofern nicht relevant, als sie erst dann zur Anwendung kommen, wenn konkrete Investitionen rechtlich abgesichert werden sollen, was bei Reallaboren eher selten der Fall ist (Drobek/ Tran 2017: 109-110). Anders sieht es bei den formellen Instrumenten des Besonderen Städtebaurechts im Hinblick auf Maßnahmen zur Sanierung, zum Umbau oder zur Erneuerung von Städten aus. Bei durchführungsorientierten Rechtsinstrumenten kann das Wissen, das durch Reallabore bzw. temporäre Interventionen produziert wird, als Entscheidungshilfe dienen. Hier kann die Kommune mehrere finanzielle, planerische und rechtliche Instrumente, zu denen auch Reallabore gehören können, kombinieren und so die Chancen erhöhen, städteräumliche und städtebauliche Vorhaben unter wirtschaftlichen, sozialen oder ökologischen Aspekten umzusetzen. Vor allem aber ermöglicht die Informelle Planung, z. B. in Form von Stadtund Quartierskonzepten, Verkehrsentwicklungsplänen oder integrierten Stadtentwicklungsansätzen, die Nutzung des Instruments Reallabor, wenn sie als fachliche Fundierung der rechtsförmlichen Planung und der politischen und administrativen Willensbildungs- und Entscheidungsprozesse betroffene Bürger/-innen in das Planungsverfahren miteinbezieht. Für die Praxis integrierter Stadt- und Quartiersentwicklung sind zudem die verschiedenen Programme der Städtebauförderung relevant. Die dort erwähnten Instrumente sind explizit informell angelegt und werden auch im Besonderen Städtebaurecht berücksichtigt. Reallabore bzw. Interventionen können gerade in diesem Zusammenhang als Instrumente eingesetzt werden, die nicht nur wie Runde Tische, Planungszellen oder Zukunftswerkstätten partizipatives und - wenn man so will - ,theoretisches ${ }^{6}$ Transformationswissen produzieren, sondern praktisches, erprobtes. Planung öffnet sich hier mit Realexperimenten dem „Ungeplanten“ (Drobek/Tran 2017: 103). Man kann also zum Schluss kommen, dass sich Instrumente und Methoden insbesondere im Hinblick auf die Partizipationsprozesse im Reallabor und in der Planung weitgehend übertragen bzw. anpassen lassen (Eckart/Ley/Häußler et al. 2018: 131). 
Tabelle 1 Unterschiede und Gemeinsamkeiten zwischen Realexperiment/Reallabor und Stadtplanung

\begin{tabular}{|c|c|c|}
\hline & Realexperiment/Reallabor (sozial-partizipativ) & Stadtplanung (partizipativ-kommunikativ) \\
\hline $\begin{array}{l}\text { Art der Wissenspro- } \\
\text { duktion }\end{array}$ & $\begin{array}{l}\text { transdisziplinär } \\
\text { reflexiv } \\
\text { ergebnisoffen } \\
\text { erklärend/transformativ } \\
\text { temporär }\end{array}$ & $\begin{array}{l}\text { praktisch-integrativ } \\
\text { begrenzt reflexiv } \\
\text { zielorientiert } \\
\text { transformativ } \\
\text { dauerhaft }\end{array}$ \\
\hline Ziele & $\begin{array}{l}\text { Nachhaltigkeit, Kohäsion, Integration als paradigmati- } \\
\text { sche Werte }\end{array}$ & $\begin{array}{l}\text { Nachhaltigkeit, Kohäsion, Integration als paradigmati- } \\
\text { sche Werte }\end{array}$ \\
\hline Wissensformen & $\begin{array}{l}\text { unsicher } \\
\text { „sozial robust“ }\end{array}$ & $\begin{array}{l}\text { unsicher } \\
\text { „sozial robust“ }\end{array}$ \\
\hline Methoden & $\begin{array}{l}\text {,klassisch` sozialwissenschaftlich } \\
\text { transdisziplinär }\end{array}$ & sozial-, technik-, planungswissenschaftlich \\
\hline Instrumente & ergebnisoffen-kollaborativ & zielorientiert-partizipativ \\
\hline Governance & kollaborativ & verhandelnd-kooperativ \\
\hline
\end{tabular}

\subsubsection{Governance}

Governance als ein konzeptionell-heuristisches Gerüst erfasst Akteure und ihre Beziehungen (hierarchische, wettbewerbliche, kooperative), die durch übergelagerte Werte und Normen geprägt werden. Im Kontext von Reallabor und Stadtplanung sind dies besonders zivilgesellschaftliche Akteure wie Anwohner/-innen, Vereine oder Initiativen, politisch-administrative Akteure wie Planungsämter oder Quartiersmanagement, politische Akteure wie Stadt- oder Bezirksräte/-rätinnen sowie wirtschaftliche Akteure wie Privateigentümer/-innen oder Wohnungsgesellschaften (Holtkamp 2007: 370-372). Dieser allgemeine Zugriff auf Governance korrespondiert mit einem ebenso allgemeinen auf Planungskultur, wenn darunter, wie erwähnt, spezifische Formen räumlicher Planung verstanden werden, die ebenfalls durch paradigmatische Denkweisen und Handlungsnormen der beteiligten Akteure in Abhängigkeit vom Kontext geprägt werden und sich in der sozialen Produktion von Räumen manifestieren (Sondermann 2017: 47). Aus der Perspektive von Governance können dann unterschiedliche Paradigmen räumlicher Planung bestimmt werden, die vom „synoptischen“ Planungsideal (rationaler Planungsansatz, intervenierende, hierarchische Governance) bis zur „diskursiven“ Planungskultur (kommunikativ-ergebnisoffener Planungsansatz, verhandelnde, kooperative Governance) reichen (Nuissl/Heinrichs 2006: 63).

Die transdisziplinäre Ausrichtung des Reallabors, der Anspruch auf Ko-Design der Realexperimente und auf KoProduktion des Wissens verlangt zunächst einmal eine möglichst breite Beteiligung von Akteuren - von zivilgesellschaftlichen ebenso wie von wirtschaftlichen, politischen oder administrativen. Eine besondere Bedeutung gewinnen dabei die unmittelbar an den (sozial-partizipativen) Realexperimenten beteiligten Anwohner/-innen und Wissenschaftler/-innen. Sie sollen - so das Selbstverständnis der Reallaborforschung - in transdisziplinäre Prozesse eingebun- den werden und auf Augenhöhe agieren. Dadurch soll gewährleistet werden, dass die Interessen und Wissensbestände der ,Praxispartner/-innen “ und die der Wissenschaftler/ -innen gleichermaßen wahrgenommen und hegemoniale Positionen vermieden werden. Das Verhältnis zu anderen Akteuren - insbesondere zum Stadtplanungsamt oder Stadtplanungsbüro - hängt von der taktischen und strategischen Ausrichtung des Reallabors ab. Prinzipiell wird es als neuer Akteur mit einem lokalen Governance-Arrangement konfrontiert, das oftmals durch etablierte Akteurstrukturen und -beziehungen gekennzeichnet ist. Inwieweit es kooperative, wettbewerbliche und hierarchische Beziehungen $\mathrm{zu}$ anderen Akteuren eingeht, hängt von den spezifischen lokalen Verhältnissen ab. In jedem Fall besitzt es in dieser Hinsicht einen erheblichen Spielraum. Im Vergleich dazu sind Planungsämter stärker in formale Verfahren eingebunden, können nicht beliebig Beteiligungsformate nutzen und kooperative, wettbewerbliche und hierarchische Beziehungen $\mathrm{zu}$ anderen Akteuren aufbauen.

Fasst man die Ergebnisse des Kapitels 2 hinsichtlich der Gemeinsamkeiten und Unterschiede von Reallabor und Stadtplanung in Bezug auf Veränderungen des urbanen Raums zusammen, so lassen sie sich wie folgt plakativ darstellen (vgl. Tabelle 1).

Im Kern laufen die vorangegangenen Bemerkungen also auf die inhaltliche (Ziele), instrumentelle (Art der Wissensproduktion, Wissensformen, Methoden, Instrumente) und akteurbezogene (Governance) Dimension von Realexperiment und Stadtplanung hinaus. In inhaltlicher Hinsicht sind beide auf Transformation angelegt und verfolgen normative Ziele. Bei der Operationalisierung und Umsetzung werden sie mit sehr konkreten bzw. praktischen Problemen konfrontiert, die sich für Realexperiment und Stadtplanung aber durchaus unterschiedlich darstellen können. In instrumenteller Hinsicht liegen beide ebenfalls nicht weit auseinander zumindest wenn man von offenen, kooperativen Planungs- 
ansätzen ausgeht -, aber auch hier kann das Realexperiment im Verhältnis zur Stadtplanung andere Perspektiven eröffnen. In akteurbezogener Hinsicht muss das Reallabor mit seinem hohen transdisziplinären Anspruch seine Position im quartierbasierten Governance-Arrangement finden, in dem sich die Stadtplanung bereits etabliert hat, wobei sich die Möglichkeit eröffnen kann, neue Konstellationen zu schaffen. Vor diesem systematisch-theoretischen Hintergrund wird im Folgenden das Verhältnis von Stadtplanung und Reallabor in der Empirie betrachtet.

\section{Explorative Fallstudie an der Schnittstelle von Freiraum- und Sozialplanung}

Die empirische Grundlage für diesen Beitrag liefert eine explorative Fallstudie zum Quartier Hannover-Sahlkamp, die im Rahmen des vom Bundesministerium für Bildung und Forschung (BMBF) geförderten Projekts „KoopLab“ seit 2018 durchgeführt wird. Das Verbundvorhaben experimentiert im Rahmen von an drei Standorten (Leipzig, Dortmund und Hannover) installierten Reallaboren mit unterschiedlichen Interventionen zur kooperativen Raumentwicklung, um wohnungsnahe Freiräume unter Beteiligung der Quartierbewohner/-innen gemeinschaftlich zu gestalten und auf diese Weise den sozialen Zusammenhalt zu stärken. Der Standort Hannover-Sahlkamp wird dabei von der Freien Universität Berlin, dem hannoverschen Stadtplanungsbüro „plan zwei“" sowie den „Internationalen StadtteilGärten Hannover e.V.“ (ISG) als Praxispartner vor Ort begleitet.

Das Quartier Sahlkamp-Mitte mit rund 5.600 Einwohnerinnen und Einwohnern in 2.500 Haushalten liegt am nordöstlichen Rand der Landeshauptstadt Hannover. Seit den 1960er-Jahren wurde es durch die öffentliche Hand errichtet, wobei der Fokus auf dem Sozialwohnungsbau lag. Seit 2009 ist das Quartier als „Stadtteil mit besonderem Entwicklungsbedarf“ Teil des Bund-Länder-Programms ,Soziale Stadt" (LHH 2015: 5). Es weicht soziodemographisch in mancherlei Hinsicht vom gesamtstädtischen und bezirksweiten Durchschnitt ab: überdurchschnittlich hoher Anteil an kinderreichen Familienhaushalten, Kindern und Jugendlichen unter 18 Jahren, an Bezieherinnen und Beziehern von sozialen Transferleistungen sowie an Deutschen mit Migrationshintergrund.

Für die hier durchgeführte Fallstudie kommen ein Mix aus ,klassischen “ sozialwissenschaftlichen Methoden und das transdisziplinäre Ko-Design und die transdisziplinäre Ko-Produktion zum Einsatz - von der Erhebung statistischer Daten, der Auswertung von Dokumenten über Interviews mit ,Expertinnen und Experten' bis zum permanenten Austausch im KoopLab-Team, Diskussionsrunden im
Quartier und laufenden Befragungen der Anwohner/-innen. Generell zeigt die Praxis, dass sich zwar abstrakt definieren lässt, was ein Realexperiment ist und welchen methodischen Anforderungen es genügen sollte, dass die empirische Umsetzung aber erhebliche, sehr praktische Herausforderungen mit sich bringt. Es gibt eben keine allgemeingültige ,Gebrauchsanweisung ' für Reallabore, sodass die Realexperimente je nach Themenfeld speziell konzipiert und auf spezielle Weise durchgeführt werden müssen, abhängig vom jeweiligen städtischen Setting.

Das Projekt „KoopLab“ ist thematisch an der Schnittstelle der inhaltlichen Handlungsfelder „Sozialer Zusammenhalt" und „Grün in der Stadt" bzw. von Freiraum- und Sozialplanung angesiedelt. Selbst wenn in der Planungspraxis - zumindest in den deutschen Kommunen - die Handlungsfelder von Freiraum- und Sozialplanung nicht zwangsläufig systematisch miteinander verknüpft werden, wird in der sozial- und planungswissenschaftlichen Debatte doch ein indirekter Zusammenhang zwischen Begegnungen in öffentlichen Freiräumen und sozialem Zusammenhalt hergestellt. In den meisten Programmen zur Stadtentwicklung werden sowohl die Einrichtung von Begegnungsorten als auch die Schaffung von Begegnungsangeboten in öffentlich zugänglichen Freiräumen gefordert und gefördert (Wiesemann 2019: 6-7). Zwar wird die Sinnhaftigkeit solcher geplanten Begegnungsangebote im Hinblick auf den sozialen Zusammenhalt ebenfalls in Frage gestellt, nichtsdestotrotz sind sie in der sozialen Quartiersentwicklung zur ,Befriedung ' der Nachbarschaften etabliert.

Vor diesem Hintergrund ist zu diskutieren, wie sinnvoll es ist, herkömmliche Verfahren der Stadtentwicklung bzw. -planung mit Ansätzen zu verbinden, die stärker die spontane, experimentelle Form der Begegnung nutzen wollen, um die soziale Kohäsion in urbanen Nachbarschaften zu verbessern. Nicht nur in Deutschland werden als neuer Ansatz Realexperimente innerhalb von Reallaboren erprobt.

Die seit 2018 in Hannover durchgeführten Realexperimente haben alle zum Ziel, die soziale Teilhabe zu fördern und den sozialen Zusammenhalt zu stärken und zwar durch die kooperative (Um-)Nutzung und Gestaltung von Freiräumen. Diese Interventionen umfassen beispielsweise das Aufstellen eines mobilen Bauwagens, der an verschiedenen Standorten im Quartier als Treffpunkt für die Nachbarschaft und der gemeinsamen Erschließung wenig genutzter Zwischenräume diente, ein Balkonkonzert in einem der ISGGärten, das einen Anlass zur Begegnung zwischen Zuhörerinnen/Zuhörern aus der direkten Nachbarschaft und entfernteren Wohngebieten schaffen sollte, oder eine „Lange Tafel“" im Stadtteilpark, bei der Anwohner/-innen mit Vertreterinnen/Vertretern des Quartiersmanagements und der Stadt Hannover Gespräche über den Park und das Stadtteilleben führten. Daneben wurden andere Formate ausprobiert 
und relativ enge Kontakte zu Projekten der Stadtgrünentwicklung und der Sozialarbeit gepflegt.

\section{Die inhaltliche Dimension: quartierbezogene Planung und transformative Reallabore}

Historisch betrachtet, machte die Hannoveraner Stadtplanung grüne Freiräume zwar bereits früh zum Thema, kümmerte sich jedoch zunächst wenig um soziale Begegnungen und sozialen Zusammenhalt. Da Sahlkamp-Mitte erst in den 1960er-Jahren in den Blick der Stadtplanung geriet (Bochynek-Friske 2001: 9-36), sind die grundlegenden städtebaulichen Ansätze charakteristisch für die damalige Zeit: Im Quartier dominieren bis zu 12-geschossige Wohnanlagen und große Mehrfamilienhäuser sowie kleine Subquartiere von Einfamilien- und Reihenhäusern. Das zeittypische Leitbild der autogerechten Stadt zeigt sich im hohen Anteil an Verkehrsflächen und das Gebiet ist geprägt durch weitläufige, monotone grüne Freiräume zwischen den Hochhäusern. Dadurch entstehen diffuse und als wenig zusammenhängend erlebbare Zwischenbereiche (Räuchle/Berding 2020: 88-89). In der ursprünglichen Konzeption des Quartiers waren zwar bestimmte Flächen explizit für Begegnungen vorgesehen, z. B. der Markt, spontane Aneignungen der Freiräume und damit deren Nutzung als Begegnungsorte waren jedoch nicht geplant.

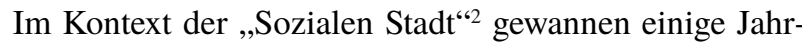
zehnte nach der baulichen Fertigstellung des Quartiers die Instandsetzung der Freiräume an Bedeutung, wobei auch ihre Aufenthaltsqualität im Vergleich zu ihrem ursprünglichen Zustand verbessert werden sollte (LHH 2013: 5). Die kommunalen Vertreter/-innen erhofften sich von einer Neugestaltung der Freiräume zugleich eine ,Befriedung' des Quartiers und einen positiven Effekt auf das soziale $\mathrm{Zu}$ sammenleben (Räuchle/Berding 2020: 89). Parallel zu den investiven Maßnahmen wurde auch verstärkt die gemeinwesenorientierte Infrastruktur mit dem Ziel ausgebaut, nicht nur soziale Netzwerke und Nachbarschaften zu stärken, sondern auch eine „Stadtteilidentität“ und eine „Kultur der Beteiligung und Mitwirkung“ zu fördern. ${ }^{3}$ Nicht mehr eine mobilitätsorientierte Nutzung der Freiflächen ist nun das

\footnotetext{
2 https://www.staedtebaufoerderung.info/StBauF/DE/ Programm/SozialeStadt/soziale_stadt_node.html (09.03.2021).

3 https://www.hannover.de/Leben-in-der-Region-Hannover/ Planen,-Bauen,-Wohnen/Stadterneuerung-Förderung/ Sanierung-im-Programm-Soziale-Stadt/Sanierung-SahlkampMitte (03.03.2021).
}

übergeordnete Leitbild bzw. Ziel, sondern eine sozial-gemeinschaftsbezogene.

Ein Reallabor wie KoopLab, das auf der Ebene des Stadtteils agiert und das normative Ziel des sozialen Zusammenhalts verfolgt, tritt damit also in ,Konkurrenz' zu einer Vielzahl an bereits etablierten europäischen, nationalen, kommunalen und quartierbezogenen Stadtplanungsprogrammen (Schneidewind 2014: 3-4). Deren normative Leitbilder weisen allerdings eine erstaunliche Kompatibilität zu Förderprogrammen auf, die reallaborbasierte Forschungen finanzieren. So zielt zum Beispiel die BMBF-Leitinitiative „Zukunftsstadt“, in deren Rahmen auch KoopLab angesiedelt ist, mit den Themen „Zuwanderung und Integration“ auf die Förderung ,partizipative[r], bürgerbestimmte[r] Forschungs- und Entwicklungsprojekte“, ${ }^{4}$ die die Bedürfnisse aller städtischen Akteure, besonders der Anwohner/-innen, und ihre soziale Vielfalt berücksichtigen, ihre „urbane Teilhabe" gewährleisten sowie Integration als Voraussetzung akzeptieren.

Nichtsdestotrotz ist gerade bei Reallaboren mit ihrer transformativen Absicht das Setzen von inhaltlichen Zielen kein unproblematisches Unterfangen, sind doch die moralischen Ansprüche an das eigene Handeln hoch. Immerhin sollen sie ein ,gesellschaftlich legitimiert[es]“ Ziel verfolgen, das ,ethisch gut begründet und gemeinwohlorientiert“ ist (Defila/Di Giulio 2018: 12). Insbesondere für ihr Zielwissen stellt sich also die Frage, aus welchen gesellschaftlichen Leitbildern diese Werte und Normen abgeleitet und wie sie gesellschaftlich legitimiert sind (Jahn/Keil 2016: 250). Das dahinterstehende demokratietheoretische bzw. -politische Problem ist zwar auch für Planungsämter und kommunal beauftragte, stadtplanerische Initiativen relevant, allerdings durch die unmittelbare Einbindung in kommunale Politikstrukturen und -prozesse nicht in gleichem Maße.

Dieser grundsätzlichen Problematik sind sich auch die am Reallabor Hannover direkt und indirekt beteiligten Akteure bewusst. Zwar identifizieren sie sich im Hinblick auf das Ziel des sozialen Zusammenhalts mit dem gesellschaftlichen Leitbild und dem Wertekanon der übergeordneten Rahmenprogrammatik und erkennen damit die Legitimation von KoopLab prinzipiell an, allerdings wird diese auch immer wieder infrage gestellt. So kam beispielsweise aus der ,organisierten Zivilgesellschaft" Kritik an den top-down gesetzten Themen (,Ich finde andere Themen viel wichtiger als Freiraumentwicklung. Nämlich einfach Wohnen“; KoopLab_LHH_15). Ein Stadtplaner reflektiert, dass das Projekt seine Legitimation auch durch das „Siegel Forschungsprojekt“ erhalte: „Das nutzen wir, um experi-

${ }^{4}$ https://www.innovationsplattform-zukunftsstadt.de/de/ zuwanderung-und-soziale-integration-1703.html (03.03.2021). 
mentelle Formate der Quartierspartizipation einzubringen ... die haben Seriosität in den Augen der Planer dadurch gewonnen, dass das nicht irgendwelche studentischen Künstleraktionen“ sind (KoopLab_LHH_17). Dennoch betonen sowohl Vertreter/-innen des Stadtplanungsamtes als auch eines freien Planungsbüros, dass sie aus der Reflexion im Kontext des Reallabors durchaus neue Einsichten und Anregungen ziehen können, z. B. zur Raumnutzung durch die Anwohnerschaft.

Das dem Reallabor-Konzept immanente Dilemma der demokratischen Legitimierung setzt sich in der Problematik fort, dass einerseits die übergeordneten Werte und Normen, die es verfolgen soll, ,paternalistisch-substanziell ' vorgegeben werden (Räuchle/Schmiz 2020: 40-41). KoopLab wird wie fast alle Reallabore durch ,politische' Drittmittel finanziert. Andererseits soll die Operationalisierung dieses normativen Zielwissens dem eigenen Selbstverständnis entsprechend ,emanzipatorisch-prozessual ' unter Mitwirkung aller Akteure formuliert werden. In einem von seiner Anwohnerschaft her sehr heterogenen Stadtteil wie HannoverSahlkamp - hier ist wieder der lokale Kontext entscheidend - ist es zudem kaum möglich, soziale Kohäsion, Partizipation oder ,gute' Nachbarschaft einvernehmlich zu bestimmen. Dies wird zum Beispiel daran deutlich, dass die im Rahmen von KoopLab befragten Anwohner/-innen Begegnungen, Kontakte und Konflikte im öffentlichen Raum des Sahlkamps sehr unterschiedlich wahrnehmen (Räuchle/ Berding 2020: 89-90). Einige Anwohner/-innen berichten von einem Gefühl der Unsicherheit. Dem steht die Erfahrung anderer Anwohner/-innen entgegen, denen im Sahlkamp „,noch nie etwas Schlimmes“ passiert sei. Diese unterschiedlichen Wahrnehmungen zum Zusammenleben im Quartier gilt es wiederum im Reallabor zum Thema zu machen.

Selbst wenn die Reallaborpraxis zeigt, dass es kaum möglich ist, den konzeptimmanenten Anforderungen gerecht zu werden und beispielsweise sozialen Zusammenhalt inhaltlich und instrumentell konsensual zu bestimmen, wird doch der Unterschied zur Stadtplanung in den kommunalen Ämtern deutlich - von performativen Ansätzen vielleicht abgesehen. Auch sie unterliegt dem normativen Dilemma, angesichts präziserer politischer Vorgaben im Rahmen der kommunalen Planung und dem geringeren Partizipationsanspruch allerdings weniger ausgeprägt. Dies mag auch für das Problem der, Übersetzung “ übergeordneter Wertvorstellungen bzw. ihrer Operationalisierung in handlungsleitende Normen gelten - eine Aufgabe, die die Stadtplanung weitgehend überfordern kann, da hier eine fortlaufende Verständigung notwendig ist.

\section{Die instrumentelle Dimension: Wissensproduktion und Realexperimente}

Das Experiment unterscheidet sich zwar in systematischtheoretischer Hinsicht von den Instrumenten konventioneller Planung, ob dies allerdings für die planerische Praxis gilt, ist durchaus umstritten und hängt vom Planungsverständnis ab. In konventionellen Planungsverfahren findet das Experiment auch in der Praxis kaum seinen Platz (Karow-Kluge 2008: 75-84). Anders sieht es aus, wenn Planungsverfahren Instrumente der experimentellen Raumproduktion nutzen. Hier betonen verschiedene an KoopLab beteiligte Akteure, dass ,dieses ganze Performative, Kollaboration, Experimentelle, Kreative, das ist überall schon da" (KoopLab_LHH_20). Die Frage sei vielmehr, inwiefern künstlerisch-kreative Ansätze in der ,normalen ' Planungspraxis bereits etabliert seien: „Ganz realistisch, in der Praxis der Planungsverwaltung ist das ein ungewöhnlicher, unüblicher Ansatz“" (KoopLab_LHH_20). Für das Verhältnis von Realexperimenten zur Stadtplanung ist auf die Überschneidung mit der Gemeinwesen- bzw. Sozialarbeit zum Beispiel im Quartiersmanagement hinzuweisen (Oehler/Drilling 2013: 28-31), nutzt diese doch seit Langem experimentelle Formate. So äußerten Mitarbeiter/-innen der Sozialarbeit in Hannover-Sahlkamp: ,...in den ersten Jahren haben wir viel rumprobiert, das muss man einfach auch mal sagen. Ich habe einfach rumprobiert und gedacht: Wie kriegst Du die Leute?" (KoopLab_LHH_13). Zumindest in der Praxis sind viele Angebote der Sozialarbeit Interventionen in Reallaboren ähnlich, mit durchaus vergleichbaren Ansprüchen an die transformativen Ziele. Zwar fehlt die wissenschaftliche Konzeption und Begleitung, verschiedene Formate können aber trotzdem als eine experimentelle Raumnutzung interpretiert werden.

Unabhängig von der Frage, ob experimentelle Instrumente in der Planungspraxis verankert sind oder nicht, schätzen die in KoopLab involvierten Akteure ihr Potenzial für Quartiere wie Sahlkamp-Mitte als relativ hoch ein, auch im Vergleich zur sonstigen hannoverschen Stadtplanung und -entwicklung und unabhängig vom konkreten Thema des Reallabors. Ein Mitarbeiter eines zivilgesellschaftlichen Vereins fasst einen experimentellen Ansatz als eine bestimmte Haltung gegenüber stadtentwicklungspolitischen Fragen generell auf: „Ich bin ein sehr großer Freund davon, dass man die Räume [in denen Planungsvorhaben durchgeführt werden sollen] erstmal wahrnimmt, ... den Raum liest. Dass man sich auf den Raum einlässt, dass man riecht, dass man schmeckt, hört, fühlt, dass man den Raum ganzheitlich wahrnimmt" (KoopLab_LHH_19). Das Realexperiment sei hier ein richtiger Ansatz. Dem lokalen Kontext kommt dabei eine entscheidende Bedeutung bei der Einschätzung zu, 
ob ein Reallabor mit seinen Realexperimenten als passendes Format für Planungsvorhaben gesehen wird. Für SahlkampMitte formuliert der Mitarbeiter: „Wir haben die Möglichkeit, über einen langen Zeitraum mit einem gewissen finanziellen und personellen Aufwand uns eines Themas anzunehmen. Wenn ich mir angucke, was in Hannover generell dazu los ist, sind das viele kurzfristigere Sachen und Beteiligungsveranstaltungen ... dieses Glück dieser drei Jahre ist für ein Quartier wie den Sahlkamp sehr gut, weil hier durch die verschiedenen Problemlagen der Menschen und diese Diversität sehr viel Vertrauensarbeit notwendig ist“ (KoopLab_LHH_19).

Dabei heben die in KoopLab involvierten Akteure immer wieder hervor, dass in Hannover auch seitens der Verwaltung eine große Offenheit gegenüber derartigen Ansätzen bestehe und somit eine kooperative lokale Planungskultur herrsche (vgl. auch Sondermann 2015: 107-108). Vertreter/-innen der Stadt Hannover betonen außerdem, dass das Reallabor mit seinen zusätzlichen Ressourcen Räume für umfassendere Partizipationsformate schaffe, die der Stadtverwaltung durch personelle und finanzielle Restriktionen verschlossen seien.

Der experimentelle Ansatz, der für Sahlkamp-Mitte passend sei, wird gleichzeitig für andere Vorhaben und Quartiere als unangemessen erachtet, beispielsweise bei einer ,Hochglanzsanierung ' in der hannoverschen Innenstadt. Gerade diesen Aspekt beurteilen aber planungspraktische und zivilgesellschaftliche Akteure wiederum kritisch: „In bürgerlichen Viertel hat man [bei Experimenten] möglicherweise mit Widerstand zu rechnen, obwohl die es genauso nötig hätten“ (KoopLab_LHH_20).

Insgesamt sind Reallabore, so auch KoopLab, mit dem methodischen Anspruch einer gleichberechtigten Beteiligung von Akteuren konfrontiert (Schneidewind 2014: 4; Wagner/Grunwald 2015: 28-30). Für KoopLab kann festgehalten werden, dass es diesem transdiziplinär-partizipativem Anspruch zumindest ansatzweise gerecht wird. Erst das längerfristige Engagement mit unterschiedlichen Interventionen führt zur Teilhabe verschiedener Anwohnergruppen. Es werden Meinungen und Wahrnehmungen zum Stadtteil sichtbar, die sonst, verborgen' bleiben würden. Beispielsweise wird deutlich, dass Kinder sich für ihr Quartier eine Skateboard-Bahn, eine Eisdiele und ein Schwimmbad wünschen, dass der Wachdienst des größten Wohnungsunternehmens meint, von Anwohner/-innen voll akzeptiert und Ansprechpartner bei Sorgen zu sein oder dass eine ältere Anwohnerin sich darüber freut, dass der Stadtteilpark nach seiner Umgestaltung so gut angenommen wird. Zwar sind nicht alle Informationen planerisch relevant und manches ist auch der Sozialarbeit bekannt, trotzdem stellt das Reallabor neues Wissen über Raumnutzungen zur Verfügung. Zugleich wird aber auch deutlich, dass der hohe Anspruch, bestehende ,Machtverhältnisse aufzubrechen und lebensweltliche Perspektiven einzubeziehen, gerade in einem superdiversen Quartier wie Sahlkamp auf nur schwer zu überwindende Barrieren stößt (Räuchle/ Schmiz 2020: 40-45).

Dennoch zeigt KoopLab, dass im Hinblick auf Transdisziplinarität und Partizipation ein Unterschied zwischen experimenteller und planerischer Wissensproduktion besteht. Das offene Realexperiment mit seiner Möglichkeit des ,Scheiterns' unterscheidet sich eben doch von den Instrumenten selbst offener Planungsverfahren wie Erörterungsveranstaltungen, Runden Tischen oder Planungsworkshops. Realexperimente dienen in erster Linie eben nicht dazu, Konflikte aufzulösen und Akzeptanz zu schaffen. Trotzdem schätzen die in KoopLab involvierten Stadtplaner/-innen ihr Potenzial als so hoch ein, dass sie dafür plädieren, diese nicht mehr nur in einer ,Nische" stattfinden zu lassen, sondern in offizielle Planungsprozesse zu integrieren bzw. diesen vorzuschalten, bevor die „eigentliche Planungsmaschinerie losgetreten wird“ (KoopLab_LHH_20). Die damit verbundenen Potenziale liegen nach Ansicht der Planer/ -innen darin, zu mobilisieren, zu aktivieren und räumliche Nutzungsmöglichkeiten aufzuzeigen (vgl. auch Altrock 2014: 24). Im Fall von KoopLab ist es jedenfalls so, dass das experimentelle Format die Voraussetzungen für soziale Kontakte bzw. Begegnungen verbessert und Anwohner/ -innen anspricht, die selbst die Beteiligungsformate einer grundsätzlich kommunikativ ausgerichteten Planungskultur nur schwer erreicht, z. B. verschiedene im Quartier ansässige migrantische Communities und Mütter mit Kindern. KoopLab macht deutlich, dass Reallabore, are a way of getting around the formal bureaucratic system in a quasiformal way, by allowing certain deviations" (Scholl/Kemp 2016: 93).

\section{Die akteurbezogene Dimension: Governance-Arrangements und Beziehungsgeflechte}

Reallabore und ihre Realexperimente können in ganz unterschiedliche Governance-Arrangements eingebettet sein von recht engen Beziehungen, in denen die Stadtverwaltung bzw. -planung unmittelbar am Reallabor beteiligt ist, bis zu recht losen, in denen das Reallabor und die Stadtverwaltung bzw. -planung nur sporadisch Kontakt haben (vgl. z. B. Scholl/Kemp 2016: 89-91; Waitz/Quint/Trenks et al. 2017: 77-78). Diese Vielfalt repräsentiert unterschiedliche Formen von Reallaboren. In KoopLab gibt es (1) das Kernteam, das für die Konzeption des Reallabors und seine Arbeit insgesamt verantwortlich ist und die verschiedenen Realexperimente entwirft und durchführt sowie die Dokumentation 
und Reflexion der Ergebnisse vornimmt. Mitarbeiter/-innen der Stadtplanung aus der hannoverschen Verwaltung gehören nicht dazu. (2) Eine weitere Gruppe von Akteuren bringen bei einzelnen Interventionen Zeit, Geld und Wissen ein. Dies gilt insbesondere für die Vertreter/-innen der sozialen Stadtentwicklung: das Quartiersmanagement und Mitarbeiter/-innen des Sachgebiets „Stadterneuerung“ innerhalb des Fachbereichs „Planen und Stadtentwicklung“ der Stadtverwaltung. Sie werden regelmäßig über die Aktivität des Reallabors informiert. Nach einer Phase der Vertrauensbildung arbeitet das Kernteam aber weitgehend unabhängig von der Stadtverwaltung bzw. -planung. Das bedeutet nicht, dass nicht weiterhin informiert und kooperiert wird. (3) Zuletzt sind in KoopLab Stakeholder und einzelne Persönlichkeiten aus Sahlkamp-Mitte involviert, die sich spontan und sporadisch an einzelnen Interventionen beteiligen. Insgesamt stellt KoopLab einen zusätzlichen Akteur in der Governance-Konstellation von Hannover-Sahlkamp dar, der gegenüber anderen Akteuren und insbesondere gegenüber der in der Kommunalverwaltung verankerten Stadtplanung relativ autonom agiert (Seebacher/Alcántara/Quint 2018: 158).

Grundsätzlich bemüht sich KoopLab aber um kooperative Beziehungen zu anderen Akteuren und versucht, an bestehende Netzwerke anzudocken. Insofern kann man es durchaus als intermediäre Schnittstelle zwischen unterschiedlichen Akteurgruppen bezeichnen. Wettbewerbliche Beziehungen um die Nutzung von Freiflächen beispielsweise zur Gemeinwesenarbeit oder zum Grünflächenamt treten nicht auf. ,Hierarchische' Beziehungen deuten sich dann an, wenn es um die Nutzung von in privatem Eigentum befindlichen Flächen geht, die nicht ohne Weiteres gewährt werden. Bei öffentlichen Flächen spielt die Dauer der Interventionen eine Rolle; eine experimentelle Raumnutzung kann dann auf Widerstand stoßen, wenn sie langfristig angelegt ist.

Geht man davon aus, dass es lokale Planungskulturen gibt, die sich in der besonderen „Interpretation von Zielen, der Anwendung von Instrumenten oder der Gestaltung von Verfahren" (Sondermann 2015: 108) ausdrücken und die wiederum in lokalspezifische Governance-Arrangements eingebettet sind, wird man die hannoversche Planungskultur als grundsätzlich ,offen ' kennzeichnen können (Sondermann 2015: 108). Kooperative, kommunikative Planung wird hier seit Längerem praktiziert, das heißt, man versucht möglichst viele Stakeholder bzw. Akteurgruppen zu beteiligen. Auch einem Reallabor wie KoopLab mit seinen Interventionen zur experimentellen Raumnutzung begegnet man zunächst einmal mit Interesse. Hannover zeigt allerdings ebenso, dass es nur bedingt eine alle Ämter übergreifende Planungskultur gibt und nicht alle uneingeschränkt einem offenen Planungsansatz mit verhandelnder, kooperativer Governance folgen. Die Zeitdimension spielt dabei eine wichtige Rolle. Bei der Entwicklung von langfris- tigen Flächennutzungsplänen ist die Governance durch das formelle Planungsverfahren festgelegt. Bei der Vergabe von Projekten an private Planungsbüros ist ein mehr oder weniger differenzierter Leistungskatalog abzuarbeiten, der ebenfalls die Beteiligung bestimmter Akteure vorschreibt, aber meist einen größeren Spielraum in dieser Hinsicht eröffnet eben auch hinsichtlich der Einbindung eines neuen Akteurs wie KoopLab. Im Übrigen besitzen die meisten Quartiere - wie auch der Sahlkamp - ausgesprochen vielschichtige Akteurkonstellationen und ausgesprochen komplexe Interessenlagen, die von formellen Planungsverfahren nur bedingt erfasst werden können, wodurch sich für ein Reallabor wie KoopLab weitergehende Beteiligungsperspektiven eröffnen.

Eine eindeutige Antwort auf die Frage, wie sich ein Reallabor im Governance-Arrangement einer Stadt bzw. eines Quartiers positionieren muss, um zielorientiert arbeiten zu können, kann es nicht geben. Dies gilt auch für ein Reallabor wie KoopLab. Reallabore, die sich ihre Autonomie bewahren, indem sie weder formelle Absichtserklärungen oder Flächennutzungsvereinbarungen mit der Stadtverwaltung abschließen noch enge Kooperationen mit den behördlichen Planungsstellen eingehen, können eventuell Anwohnergruppen, die den kommunalen Akteuren wenig Vertrauen entgegenbringen, eher einbinden als solche, die sehr eng mit der Verwaltung zusammenarbeiten. Informelle, lose Beziehungen können insofern für Reallabore, die das Ziel verfolgen, über die Stärkung von Partizipation und Empowerment die soziale Kohäsion im Quartier zu fördern, als experimentelle Nische im vorgefundenen Governance-Arrangement durchaus erfolgversprechend sein. Ein Reallabor kann allerdings aus strategischen Gründen engere Kooperationsbeziehungen zu potenten Akteuren suchen, um ihre Position gegenüber den Planungsämtern zu stärken, so z. B. zu den vor Ort aktiven Wohnungsgesellschaften. Es riskiert dann allerdings, die Beziehungen zu anderen Akteuren zu belasten. Neben solchen kooperativen Beziehungen können auch wettbewerbliche auftreten, wenn das Reallabor Interventionen ins Auge fasst, die ebenfalls von der Gemeinwesen- bzw. Quartiersarbeit geplant sind oder durchgeführt werden, was bei KoopLab, wie erwähnt, nicht der Fall ist. Ob das innovative Potenzial eines Reallabors von der Stadtplanung, vielleicht auch gegen sie genutzt wird, hängt jedenfalls nicht von diesen beiden Akteuren allein ab, sondern vom lokalen, quartierbezogenen Governance-Arrangement insgesamt. Das Reallabor bietet nach den Erfahrungen von KoopLab aber die Chance, intensivere Kontakte zwischen den verschiedenen Akteuren herbeizuführen, formalisierte Planungsverfahren zu öffnen und die Teilhabe zu erweitern. 


\section{Reallabore als potenzielle Chancen für Stadtplanung}

Zum Schluss soll die anfangs gestellte Frage noch einmal aufgegriffen werden: Welche Chancen kann das Reallabor bzw. Realexperiment der Stadtplanung bieten? Der thematische Ausgangspunkt bei der Beantwortung dieser Frage ist die kooperative Freiraumgestaltung zur Stärkung sozialer Kohäsion, der methodische Ansatzpunkt die experimentelle Wissensproduktion und der transdisziplinär-transformative Ansatz des Reallabors, der organisatorische Ansatzpunkt ist KoopLab, der lokale Ansatzpunkt das superdiverse, migrationsgeprägte Ankunftsquartier Hannover-Sahlkamp. Inwieweit solche fallstudienbasierten Erfahrungen verallgemeinerbar sind, ob die potenziellen Chancen von der Stadtplanung aufgegriffen werden und eventuell sogar zu institutionellen Veränderungen führen, ist auch in der Reallaborforschung noch nicht endgültig beantwortet. Dies hängt davon ab, welches Ziel bzw. Thema das Reallabor verfolgt, wie es organisiert ist und in welchem lokalen Kontext es agiert, insbesondere welche Planungskultur vor Ort herrscht. Mit der abschließenden Nennung der potenziellen Chancen soll im Übrigen nicht der Eindruck erweckt werden - dies sei noch einmal betont -, dass die Art und Weise der experimentellen Wissensproduktion des Reallabors für die Planungswissenschaft und -praxis etwas völlig Neues wäre. Vielmehr greift das Reallabor-Konzept in mancherlei Hinsicht die (performativen) Ansätze auf, die sich bereits in der Planungswissenschaft und -praxis in der jüngeren Vergangenheit herausgebildet haben, sodass sich ,nur' die Frage stellt, inwiefern Realexperimente bekannte Planungsinstrumente sinnvoll ergänzen können.

\subsection{Inhaltliche Dimension}

Zunächst scheint das Reallabor mit seinen experimentellen, transdisziplinären, niedrigschwelligen Interventionen das Verhandeln von übergeordneten Werten und deren praxisbezogener Operationalisierung im quartierbezogenen Kontext auf eine Weise zu ermöglichen, die auch Meinungen und Wahrnehmungen berücksichtigt, die im formalen, offiziellen Planungsverfahren nur bedingt Beachtung finden. Vor diesem Hintergrund können Realexperimente in der Stadtplanung, besonders wenn sie den eigentlichen Planungsprozessen vorgeschaltet sind, alternative Formen der anwohnerbezogenen Aneignung von urbanen Räumen und neue Ideen der Raumproduktion aufzeigen. Insofern kann durch Reallabore eventuell ein differenzierteres Bild des Diskurses über ein geplantes Vorhaben gezeichnet werden. Auch die theoretische Reflexion des im Reallabor gewonnenen Wissens kann den planerischen Transformationszielen Impulse geben. Das konkrete Planungsvorhaben und die herrschende Planungskultur entscheiden darüber, ob diese Chancen genutzt werden. Zudem können Reallabore und die in ihnen stattfindenden Realexperimente eine stärkere Identifikation mit dem Quartier schaffen - weniger nach außen, dies kann die konventionelle Planung auch, sondern mehr nach innen.

\subsection{Instrumentelle Dimension}

Reallabore können die Teilhabe der Anwohner/-innen an der Planung quartierbezogener Projekte erweitern. Ihr transdisziplinärer Anspruch - eventuell verbunden mit einer gezielten Strategie des Empowerments -, ihre längerfristige Perspektive, ihre unterschiedlichen Realexperimente mit den kollaborativen, informellen Angeboten zur Beteiligung bieten das Potenzial, für die Stadtplanung nur schwer erreichbare Gruppen der Anwohnerschaft zu adressieren. Vor allem für sozial-partizipative Vorhaben kann eine erweiterte Einbeziehung der Anwohner/-innen Vorteile für den Planungsprozess und das Erreichen des Planungsziels bringen. Das von ihnen genutzte Format des Realexperiments, ihre ,Neutralität' im Sinne einer möglichen Distanz zu anderen Akteuren - insbesondere zu Planungsämtern oder zu Wohnungsgesellschaften - und ihre mögliche Nähe zu den Anwohnerinnen und Anwohnern sowie wiederum ihr längerfristiges Engagement vor Ort versetzen sie in die Lage, Vertrauen aufzubauen, was den Planungsämtern nur bedingt gelingen kann. Eine Chance von Reallaboren liegt weiterhin darin, dass diese wie die Planungsvorhaben zwar ein transformatives, normatives Ziel verfolgen, ihre Realexperimente aber weniger darauf ausgerichtet sind, die Voraussetzungen zur Durchsetzung dieses Ziels zu schaffen. Sie dienen vielmehr primär einer offenen Wissensproduktion, lassen ,Scheitern' zu und sind auf Reflexion des gewonnenen Wissens hin angelegt. Experimentiert werden kann mit unterschiedlichen (Beteiligungs-)Formaten, was im Rahmen der Planung kaum möglich ist. Offenheit, Reflexion und ,emphatisches Verständnis' für lokale Belange kommen in konventionellen Planungsverfahren ebenfalls oftmals zu kurz, können aber zumindest für eine offene Planungskultur hilfreich sein, eventuell als Vorstufe zum eigentlichen Planungsprozess. Zudem bringen Reallabore eigene Ressourcen mit: Zeit, Geld und Personal. Selbst wenn die Sinnhaftigkeit oder der Mehrwert von Realexperimenten bzw. ,experimenteller Planung ' von der offiziellen Stadtplanung nicht bestritten wird, kann es ihr doch an zeitlichen, personellen und finanziellen Ressourcen fehlen. All diese werden ihr durch das Reallabor quasi umsonst zur Verfügung gestellt. Daraus folgt unmittelbar die Frage, inwieweit Reallabore kurzfristig Aufgaben einer partizipativen Planung der Kommunalverwaltung übernehmen oder Anstöße für langfristige institutionalisierte Verfahren in der Planung geben können. 


\subsection{Akteurbezogene Dimension}

Das Reallabor betritt als neuer Akteur das lokale, quartierbezogene Governance-Arrangement mit seinen etablierten Akteurstrukturen und -beziehungen. Wiederum gilt auch hier, dass es solche Anwohner/-innen erreichen kann, die von der Planung - aus welchen Gründen auch immer - nicht erreicht werden. Insofern können Reallabore als intermediäre Schnittstellen zwischen unterschiedlichen Akteurgruppen agieren. Sie können an bestehende Netzwerke andocken, Akteure zusammenführen, die bisher wenig Kontakt miteinander hatten, oder flexible Formate der Zusammenarbeit einrichten, wozu die Stadtplanung im Rahmen ihrer formalen Planungsverfahren nicht zwangsläufig in der Lage ist - aufgrund rechtlich oder bürokratisch festgelegter Beteiligungsformen, mangelnder Zeit oder fehlender personeller oder finanzieller Mittel. Wenn partizipative, deliberative Beteiligung ein Ziel von Planung ist, kann diese durch Reallabore gestärkt werden. Eine zentrale Voraussetzung dafür, dass das Realexperiment als zusätzliches Instrument der Stadtplanung genutzt wird, ist die vertrauensvolle Zusammenarbeit zwischen Reallabor und Planungsämtern bzw. Planungsbüros.

In diesem Beitrag wurde argumentiert, dass Realexperimente und Stadtplanung - zumindest in bestimmten Varianten - eine gewisse Kompatibilität aufweisen und erstere ein Potenzial als ergänzendes Instrument besitzen. In zukünftigen Forschungen können vergleichende Analysen von Reallaboren helfen zu verstehen, welchen Einfluss lokale, institutionelle Kontexte auf dieses Potenzial haben. Interessant wäre auch eine Typenbildung von Reallaboren hinsichtlich der Binnenstruktur, der verwendeten Instrumente und der Einbindung in das lokale Governance-Arrangement, um daraus Rückschlüsse auf die Potenziale ziehen zu können. Im Vergleich zu technisch-ökologischen Reallaboren sind Wirkungsanalysen bei sozial-partizipativen weitestgehend unterentwickelt. Hier besteht ebenfalls ein großer Forschungsbedarf.

\section{Verzeichnis der zitierten Interviews}

\begin{tabular}{|c|c|c|}
\hline Akteursgruppe & Interview-Code & $\begin{array}{l}\text { Interview- } \\
\text { Datum }\end{array}$ \\
\hline $\begin{array}{l}\text { Stadtverwaltung } \\
\text { (u.a. Planungsamt, Quar- } \\
\text { tiersmanagement, Gemein- } \\
\text { wesenarbeit) }\end{array}$ & KoopLab_LHH_13 & 15.01 .2020 \\
\hline $\begin{array}{l}\text { Zivilgesellschaftliche Akteure } \\
\text { (u.a. Vereine, Bildungsein- } \\
\text { richtungen, lokale Initiati- } \\
\text { ven, Einzelpersonen) }\end{array}$ & $\begin{array}{l}\text { KoopLab_LHH_15 } \\
\text { KoopLab_LHH_19 }\end{array}$ & $\begin{array}{l}15.01 .2020 \\
24.09 .2020\end{array}$ \\
\hline $\begin{array}{l}\text { Wirtschaft } \\
\text { (Planungsbüros, Wohnungs- } \\
\text { unternehmen) }\end{array}$ & $\begin{array}{l}\text { KoopLab_LHH_17 } \\
\text { KoopLab_LHH_20 }\end{array}$ & $\begin{array}{l}29.01 .2020 \\
29.09 .2020\end{array}$ \\
\hline
\end{tabular}

Förderhinweis Diese Open-Access-Publikation wurde durch den $\mathrm{Pu}-$ blikationsfonds der Freien Universität Berlin gefördert.

\section{Literatur}

Abbott, J. (2005): Understanding and managing the unknown. The nature of uncertainty in planning. In: Journal of Planning Education and Research 24, 3, 237-251. https://doi.org/10.1177/0739456X04267710

Altrock, U. (2014): Das Ende der Angebotsplanung? Instrumente der Planung im Wandel. In: Küpper, P.; Levin-Keitel, M.; Maus, F.; Müller, P.; Reimann, S.; Sondermann, M.; Stock, K.; Wiegand, T. (Hrsg.): Raumentwicklung 3.0 - Gemeinsam die Zukunft der räumlichen Planung gestalten. Hannover, 15-32. = Arbeitsberichte der ARL 8.

Beecroft, R.; Trenks, H.; Rhodius, R.; Benighaus, C.; Parodi, O. (2018): Reallabore als Rahmen transformativer und transdisziplinärer Forschung. Ziele und Designprinzipien. In: Defila, R.; Di Guilio, A. (Hrsg.): Transdisziplinär und transformativ forschen. Eine Methodensammlung. Wiesbaden, 75-100. https://doi.org/10.1007/9783-658-21530-9_4

Bochynek-Friske, A. (2001): Ein Stadtteil von Hannover. Der Sahlkamp, Unser Zuhause. Hannover.

Caprotti, F.; Cowley, R. (2017): Interrogating urban experiments. In: Urban Geography 38, 9, 1441-1450. https:// doi.org/10.1080/02723638.2016.1265870

Danielzyk, R.; Sondermann, M. (2018): Informelle Planung. In: Akademie für Raumforschung und Landesplanung (Hrsg.): Handwörterbuch der Stadt- und Raumentwicklung. Hannover, 963-974.

Defila, R.; Di Giulio, A. (2018): Reallabore als Quelle für die Methodik transdisziplinären und transformativen Forschens - eine Einführung. In: Defila, R.; Di Giulio, A. (Hrsg.): Transdisziplinär und transformativ forschen. 
Eine Methodensammlung, Wiesbaden, 9-35. https://doi. org/10.1007/978-3-658-21530-9_1

Defila, R.; Di Giulio, A. (2019): Wie Reallabore für Herausforderungen und Expertise in der Gestaltung transdisziplinären und transformativen Forschens sensibilisieren - eine Einführung. In: Defila, R.; Di Giulio, A. (Hrsg.): Transdisziplinär und transformativ forschen, Band 2. Eine Methodensammlung, Wiesbaden, 1-30. https://doi. org/10.1007/978-3-658-27135-0_1

Deutscher Städtetag (2013): Integrierte Stadtentwicklungsplanung und Stadtentwicklungsmanagement - Strategien und Instrumente nachhaltiger Stadtentwicklung. Berlin.

Dorstewitz, P. (2014): Planning and experimental knowledge production: Zeche Zollverein as an urban laboratory. In: International Journal of Urban and Regional Research 38, 2, 431-449. https://doi.org/10.1111/14682427.12078

Drobek, S.; Tran, M.-C. (2017): Temporäre urbane Interventionen in der Stadtplanungspraxis. In: Reinermann, J.-L.; Behr, F. (Hrsg.): Die Experimentalstadt. Kreativität und die kulturelle Dimension der Nachhaltigen Entwicklung. Wiesbaden, 95-114. https://doi.org/10.1007/978-3-65814981-9_6

Eckart, J.; Ley, A.; Häußler, E.; Erl, T. (2018): Leitfragen für die Gestaltung von Partizipationsprozessen in Reallaboren. In: Defila, R.; Di Giulio, A. (Hrsg.): Transdisziplinär und transformativ forschen. Eine Methodensammlung. Wiesbaden, 105-135. https://doi.org/10.1007/9783-658-21530-9_6

Evans, J.; Karvonen, A.; Raven, R. (2016): The experimental city: new modes and prospects of urban transformation. In: Evans, J.; Karvonen, A.; Raven, R. (Hrsg.): The experimental city. London, 1-12.

Fürst, D. (2018): Planung. In: Akademie für Raumforschung und Landesplanung (Hrsg.): Handwörterbuch der Stadt- und Raumentwicklung. Hannover, 1711-1719.

Groß, M. (2017): Experimentelle Kultur und die Governance des Nichtwissens. In: Reinermann, J.-L.; Behr, F. (Hrsg.): Die Experimentalstadt. Kreativität und die kulturelle Dimension der Nachhaltigen Entwicklung. Wiesbaden, 21-40. https://doi.org/10.1007/978-3-65814981-9_2

Hahne, U. (2019): Reallabore. Katalysator für die nachhaltige Stadt- und Regionalplanung? In: Raumplanung 200, 1, 50-55.

Holtkamp, L. (2007): Local Governance. In: Benz, A.; Lütz, S.; Schimank, U.; Simonis, G. (Hrsg.): Handbuch Governance. Theoretische Grundlagen und empirische Anwendungsfelder. Wiesbaden, 366-377. https://doi.org/10. 1007/978-3-531-90407-8_27

Honey-Rosés, J.; Stevens, M. (2019): Commentary on the absence of experiments in planning. In: Journal of Plan- ning Education and Research 39, 3, 267-272. https://doi. org/10.1177/0739456X17739352

Jacobsen, S. (2018): Challenging the place experiment. A critical take on the experiment as an urban planning strategy. Masterthesis an der Universität Lund.

Jahn, T.; Keil, F. (2016): Reallabore im Kontext transdisziplinärer Forschung. In: GAIA - Ecological Perspectives for Science and Society 25, 4, 247-252. https://doi.org/ 10.14512/gaia.25.4.6

Kanning, H. (2018): Reallabore aus planerischer Perspektive. Hannover. = sustainify Arbeits- und Diskussionspapier 3.

Karow-Kluge, D. (2008): Gewagte Räume. Experimente als Teil von Planung zwischen Wissenschaft, Gesellschaft und Kunst. Dissertation an der Leibniz Universität Hannover.

Karvonen, A.; van Heur, B. (2014): Urban laboratories: Experiments in reworking cities. In: International Journal of Urban and Regional Research 38, 2, 379-392. https:// doi.org/10.1111/1468-2427.12075

Knieling, J. (2018): Kooperative Planung. In: Akademie für Raumforschung und Landesplanung (Hrsg.): Handwörterbuch der Stadt- und Raumentwicklung. Hannover, 1229-1236.

LHH - Landeshauptstadt Hannover, Fachbereich Planen und Stadtentwicklung (2013): Freiraumentwicklungskonzept. Dokumentation. Hannover.

LHH - Landeshauptstadt Hannover, Fachbereich Planen und Stadtentwicklung (2015): Integriertes Entwicklungskonzept 2015 Sahlkamp-Mitte. Sanierung ,Soziale Stadt". Hannover.

May, T.; Perry, B. (2016): Cities, experiments and the logics of the knowledge economy. In: Evans, J.; Karvonen, J.; Raven, R. (Hrsg.): The Experimental City. London, 3246.

Nuissl, H.; Heinrichs, D. (2006): Zwischen Paradigma und heißer Luft: Der Begriff der Governance als Anregung für die räumliche Planung. In: Altrock, U.; Güntner, S.; Huning, S.; Kuder, T.; Nuissl, H.; Peters, D. (Hrsg.): Sparsamer Staat - Schwache Stadt? Berlin, 51-72. = Planungsrundschau 13.

Oehler, P.; Drilling, M. (2013): Soziale Arbeit, Gemeinwesenarbeit und Stadtentwicklung. Eine theoriegeschichtliche Spurensuche. In: Drilling, M.; Oehler, P. (Hrsg.): Soziale Arbeit und Stadtentwicklung. Forschungsperspektiven, Handlungsfelder, Herausforderungen. Wiesbaden, 13-41. https://doi.org/10.1007/978-3-658-10932-5_1

Othengrafen, F.; Reimer, M. (2018): Planungskultur. In: Akademie für Raumforschung und Landesplanung (Hrsg.): Handwörterbuch der Stadt- und Raumentwicklung. Hannover, 1733-1739. 
Parodi, O.; Beecroft, R.; Albiez, M.; Quint, A.; Seebacher, A.; Tamm, K.; Waitz, C. (2016): Von „Aktionsforschung“ bis „Zielkonflikte“. Schlüsselbegriffe der Reallaborforschung. In: Technikfolgenabschätzung - Theorie und Praxis 25, 3, 9-18. https://doi.org/10.14512/tatup. 25.3.9

ProClim - Forum für Klima und Global Change (1997): Forschung zu Nachhaltigkeit und Globalem Wandel - Wissenschaftspolitische Visionen der Schweizer Forschenden. Bern.

Puttrowait, E.; Dietz, R.; Gantert, M.; Heynold, J. (2018): Der Weg zum Realexperiment - Schlüsselakteure identifizieren, Kooperationsstrukturen aufbauen, Projektideen auswählen. In: Defila, R.; Di Giulio, A. (Hrsg.): Transdisziplinär und transformativ forschen. Eine Methodensammlung. Wiesbaden, 195-232. https://doi.org/ 10.1007/978-3-658-21530-9_11

Räuchle, C.; Berding, U. (2020): Freiräume als Orte der Begegnung. Planerische Leitbilder und allägliche Erfahrungen in superdiversen Stadtquartieren. In: Standort - Zeitschrift für Angewandte Geographie 44, 1, 86-92. https:// doi.org/10.1007/s00548-020-00646-X

Räuchle, C.; Schmiz, A. (2020): Wissen Macht Stadt: Wie in Reallaboren Stadt verhandelt und Wissen produziert wird. In sublurban - zeitschrift für kritische stadtforschung 8, 3, 31-52.

Schäfers, B. (1992): Planung. In: Schäfers, B. (Hrsg.): Grundbegriffe der Soziologie. Opladen, 232-234.

Schäpke, N.; Stelzer, F.; Bergmann, M.; Singer-Brodowski, M.; Wanner, M.; Caniglia, G.; Lang, D. J. (2017): Reallabore im Kontext transformativer Forschung. Ansatzpunkte zur Konzeption und Einbettung in den internationalen Forschungsstand. Lüneburg. = IETSR Discussion Papers in Transdisciplinary Sustainability Research 1/2017.
Schneidewind, U. (2014): Urbane Reallabore - ein Blick in die aktuelle Forschungswerkstatt. In: PND Online 3, 1-7.

Scholl, C.; Kemp, R. (2016): City labs as vehicles for innovation in urban planning processes. In: Urban Planning 1, 4, 89-102. https://doi.org/10.17645/up.v1i4.749

Seebacher, A.; Alcántara, S.; Quint, A. (2018): Akteure in Reallaboren - Reallabore als Akteure. In: Defila, R.; Di Giulio, A. (Hrsg.): Transdisziplinär und transformativ forschen. Eine Methodensammlung. Wiesbaden, 155159. https://doi.org/10.1007/978-3-658-21530-9_9

Sondermann, M. (2015): Zivilgesellschaftliches Engagement und die kulturelle Dimension kooperativer Stadtgrünentwicklung am Beispiel Hannovers. In: Neues Archiv für Niedersachsen 1, 98-111.

Sondermann, M. (2017): Planungskultur als Sinnsystem. Eine Untersuchung am Beispiel kooperativer Stadtgrünentwicklung in Düsseldorf. In: Raumforschung und Raumordnung | Spatial Research and Planning 75, 1, 45-56. https://doi.org/10.1007/s13147-016-0460-1

Wagner, F.; Grunwald, A. (2015): Reallabore als Forschungs- und Transformationsinstrument. Die Quadratur des hermeneutischen Zirkels. In: GAIA - Ecological Perspectives for Science and Society 24, 1, 26-31. https:// doi.org/10.14512/gaia.24.1.7

Waitz, C.; Quint, A.; Trenks, H.; Parodi, O.; Jäkel, A.; Lezuo, D.; Wäsche, H. (2017): Das Reallabor als Motor für nachhaltige Quartiersentwicklung - Erfahrungen aus dem Karlsruher Experimentierraum. In: Berichte. Geographie und Landeskunde 91, 1, 67-80.

Wiesemann, L. (2019): Begegnung schaffen im Quartier. Eine Reflexion von Theorie und Praxis. Berlin. = vhwwerkSTADT 34. 